\title{
LHomme
}

L'HOMME Revue française d'anthropologie

187-188 | 2008

Miroirs transatlantiques

\section{Cherry Schrecker, La Communauté. Histoire critique d'un concept dans la sociologie anglo-saxonne}

Samuel Lézé

\section{OpenEdition}

1 Journals

Édition électronique

URL : https://journals.openedition.org/lhomme/20612

DOI : 10.4000/lhomme.20612

ISSN : 1953-8103

Éditeur

Éditions de l'EHESS

\section{Édition imprimée}

Date de publication : 3 octobre 2008

Pagination : 482-483

ISBN : 978-2-7132-2186-6

ISSN : 0439-4216

\section{Référence électronique}

Samuel Lézé, « Cherry Schrecker, La Communauté. Histoire critique d'un concept dans la sociologie anglosaxonne ", L'Homme [En ligne], 187-188 | 2008, mis en ligne le 16 décembre 2008, consulté le 24 avril 2022. URL : http://journals.openedition.org//homme/20612; DOI : https://doi.org/10.4000//homme. 20612

Ce document a été généré automatiquement le 24 avril 2022.

(c) École des hautes études en sciences sociales 


\title{
Cherry Schrecker, La Communauté. Histoire critique d'un concept dans la sociologie anglo-saxonne
}

\author{
Samuel Lézé
}

\section{RÉFÉRENCE}

Cherry SCHRECKER, La Communauté. Histoire critique d'un concept dans la sociologie anglosaxonne. Paris, L'Harmattan, 2006, 283 p. (« Logiques sociales »).

1 AVEC PLUS DE 86 acceptions déjà répertoriées ${ }^{1}$, la notion de communauté n'a rien à envier à celle de culture ${ }^{2}$, synonyme dans certains contextes, et qui a connu à bien des égards une fortune semblable. Il en va de même pour les succès scientifiques et mondains $d^{\prime}$ ' identité $»^{3}$ ou de «tradition $»^{4}$. Comment un concept peut-il imposer l'illusion d'une telle transparence?

2 C'est à cette question que Cherry Schrecker s'attelle en procédant à un travail élémentaire, et pourtant fort salutaire pour les sciences sociales, de critique logique et lexicologique du concept de "communauté » afin d'en expliciter les présupposés. À partir d'un inventaire systématique qui rassemble un corpus conséquent de recherches sociologiques anglo-saxonnes plus ou moins cohérentes s'échelonnant entre 1918 et 2003 - les community studies -, l'auteur analyse la normativité spontanée, véhiculée par son halo sémantique. En paraphrasant une étude célèbre de Georges Canguilhem consacrée au concept de cellule ${ }^{5}$, il est possible de dire que les valeurs affectives et sociales de solidarité, de chaleur, d'intimité et d'autonomie planent sur le développement de la théorie des communautés. Le concept bénéficie donc d'une considérable surdétermination affective.

3 Ainsi, dans la première partie de son étude, Cherry Schrecker reconstruit une histoire à partir de la catégorie de totalité qui naturalise depuis Aristote le lien social (chap. I). Si ce chapitre tracé à grands traits ne rend pas complètement compte des filiations 
oubliées, recouvrements occultés et chemins brouillés du concept, l'essentiel est présent : le stagirite avait une conception organiciste et téléologique de l'organisa- tion sociale qui s'est transmise, à partir d'exemples clés et de proche en proche, à Durkheim, Tönnies, Weber, etc. Un concept prend tout son sens lorsqu'on ouvre le problème qu'il tente de résoudre et d'archiver. L'auteure en dégage deux: penser l'intégration de l'individu à la société, penser la continuité dans la transformation. Dans ce contexte, la communauté s'oppose à la société. Il s'agit au fond de réduire la modernité à une simple altération en rechignant à en déceler la positivité (e.g. l'émancipation de l'autorité traditionnelle) tant l'analogie de la totalité-solidarité organique devient nostalgie des liens communautaires. De ce fait, cette présentation aurait beaucoup gagné à être replacée, même sommairement, dans le contexte académique américain des études sociologiques sur le stress qui forme comme le contrepoint sociologique ou le négatif de cette attention à la communauté, la société (ou la "vie moderne » qui déracine et atomise) étant le lieu de développement de tous les fléaux contemporains: de la dépression à la délinquance juvénile, etc. De même, les usages sociaux, idéologiques et politiques de la "communauté » en tant que solution aux problèmes sociaux auraient mérité d'être esquissés ${ }^{6}$.

4 Le terme agit comme une bonne image qui survalorise l'appartenance locale dans une conjoncture de bouleversement social. Figure du consensus paisible et béat, la communauté ignorerait les conflits. De ce fait, la méthodologie, de préférence employée par ces "sociologues", est le travail de terrain et la référence à l'anthropologie sociale est très marquée par la quête des relations authentiques (chap. II). À cet égard, il faut peut-être souligner encore plus fortement que l'auteure elle-même, dans ce chapitre et le suivant, la forte présence d'anthropologues sous cette étiquette qui offre, à un moment charnière de l'histoire de l'anthropologie (les années 1960), la possibilité de reconvertir les routines méthodologiques ou théoriques du culturalisme comme du fonctionnalisme ${ }^{7}$. La "communauté » fonctionne comme un concept frontière opérant à peu de frais la transposition de questions anthropologiques et facilitant les analogies avec les cultures, unités d'analyses enrichies des mêmes propriétés sociales idéalisées (cohérence, consensus, stabilité, chaleur, authenticité, etc.). Se trouve alors tout simplement escamotée la réalité moins attrayante des petits groupes sociaux faits de tensions, de contrôle social, de conformisme et d'inégalité...

5 Les deuxième et troisième parties qui représentent l'essentiel de l'ouvrage dressent un tableau très didactique des études phares sur la communauté qui restent malgré tout, faute de traduction ou de compréhension de ce contexte, très mal connues en France. Il s'agit donc d'une introduction aux community studies. Le critère de spatialité et la taille de l'unité d'analyse sont retenus par Cherry Schrecker pour classer l'essentiel de cette littérature prolifique, parfois devenue célèbre: se succèdent les présentations de recherches menées sur les villages et isolats (e.g. la thèse de doctorat d'Erving Goffman : 96-101), les petites villes (e.g. W. Loyd Warner et ses Yankee City Series) et quartiers de grandes villes (e.g. William F. Whyte et son Street Corner Society). Puis, dans un second temps, sont exposées des études sur le maintien des liens communautaires (Thomas et Znaniecki, The Polish Peasant in Europe and America), les communautés de métier (camionneurs, marins-pêcheurs, etc.), ou plus récemment encore, les communautés virtuelles (les jeux d'aventure).

6 Le sort épistémologique qu'il faut réserver à ce genre de concept hyperbolique n'est pas, sous la plume de l'auteure, formulé très clairement : est-il périmé ou conserve- $t$-il 
une part de fécondité? En rectifier la portée ou injecter une part de dynamisme (historicité, conflictualité, changement d'échelle, etc. des groupes sociaux) suffit-il pour en neutraliser les présupposés? Je crains que non. L'essentiel n'est pas dans le concept (ou plutôt dans la prénotion savante), mais dans la problématique qu'il tente, un peu trop rapidement, d'étouffer en accumulant les enquêtes empiriques et les évidences. Dès lors, les études de communauté sont une solution simple à des problèmes sociaux ou sociologiques subtils et profonds qui relèvent le plus souvent de plusieurs processus sociaux imbriqués qu'il est nécessaire de distinguer8. Aussi, est-ce en réactivant la problématique et en ouvrant les diverses lignes de recherche qu'elles recèlent qu'il faut escompter un progrès sociologique. Un édifice théorique exige en effet de solides refondations et non, comme le montrent certaines tentatives de sauvetage, un simple ravalement de façade. Abandonnons donc ce concept, sans remords.

\section{NOTES}

1. G. A. Jr. Hillery, «Definitions of Community: areas of agreement», Rural Sociology 1955, 20 (1): 111-123.

2. A. L. Kroeber \& C. Kluckhohn, Culture. A Cri-tical Review of Concepts and Definitions, Cambridge, Harvard University Press, 1952.

3. Martina Avanza \& Gilles Laferté, «Dépasser la "construction des identités"? Identification, image sociale, appartenance», Genèses, 2005, 61: 154-167.

4. Eric Hobsbawm \& Terence Ranger, L'Invention de la tradition, Paris, Amsterdam, 2006.

5. «Avec la cellule, nous sommes en présence d'un objet biologique dont la surdétermination affective est incontestable et considérable [...]. Qui sait si en empruntant consciemment à la ruche des abeilles le terme de cellule pour désigner l'élément de l'organisme vivant, l'esprit humain ne lui a pas emprunté aussi, presque inconsciemment, la notion de travail coopératif? [...] Des valeurs affectives et sociales de coopération et d'association planent sur le développement de la théorie cellulaire» (La Connaissance de la vie, Paris Presses universitaires de France, 1992 [1965]: 48-49).

6. À cet égard, un article déjà ancien de Bernard Lacroix aurait pu servir de fil directeur : «Le discours communautaire», Revue française de science politique, 1974, 24 (3): 526-558.

7. Sur ce point, voir en particulier Daniel Céfaï, L'Enquête de terrain, Paris, La Découverte, 2003 [Cf. mon compte rendu dans L'Homme, 2005, 175-176: 487-490].

8. C'est le sens, par exemple, de l'article de Martina Avanza et Gilles Laferté déjà cité. 